\title{
Histoire et expérience fondatrice dans Sein und Zeit
}

Par AURELIEN ZINCQ

FNRS - Université de Liège

\begin{abstract}
Il est toutefois une expérience immédiate, et accessible à tous, qui permettrait de fonder une nouvelle conception du temps.

Un ancien mythe de l'Occident fait de cette expérience la patrie originelle de l'homme, tant elle est essentielle à l'humain. Il s'agit du plaisir' ${ }^{1}$.
\end{abstract}

\section{Introduction}

La question du sens de l'Être est irrémédiablement liée à celle de l'accès à une existence authentique ${ }^{2}$. Cette conversion à l'authenticité, qui signifie le rejet du mode d'existence impropre dans le «on»- le discours ambiant social et idéologique ${ }^{3}$-, est le résultat d'une expérience fondatrice, dont l'analytique existentiale qui se déploie dans Sein und Zeit a pour mission d'indiquer la nature. La réponse à la question du sens de l'Être ne peut advenir qu'à la condition qu'une telle expérience soit vécue : la résolution du problème de la métaphysique occidentale ne revêt donc pas un caractère cognitif - elle n'est pas un problème de connaissance -, elle concerne notre capacité à vivre une expérience d'un type bien particulier.

${ }^{1}$ Giorgio Agamben, Enfance et histoire, trad. fr. Y. Hersant, Paris, Éditions Payot, coll. « Petite Bibliothèque Payot», 2010, p. 177.

2 J'écris « Être » lorsqu'il s'agit de l'être de l'étant, de l'être en totalité ou de l'étantité de l'étant; j'écris "être» lorsqu'il s'agit d'indiquer l'être de celui qui, dans l'horizon du temps, existe en ayant rapport à l'Être (cf. Michel Haar, Heidegger et l'essence de l'homme, Grenoble, Éditions J. Millon, coll. « Krisis », 2002, p. 8).

${ }^{3}$ Marc Richir, Du sublime en politique, Paris, Éditions Payot, coll. « Critique de la Politique », 1991, p. 357. 
Dans cette étude, je me propose de parcourir Sein und Zeit pour tenter de déterminer quelle pourrait être cette expérience fondatrice qui doit mener le Dasein vers son existence authentique. Après quelques remarques d'ordre général sur l'analytique existentiale, je me focaliserai sur les paragraphes de Sein und Zeit qui sont spécifiquement consacrés à l'historialité. Je pense en effet que c'est à l'intérieur de ceux-ci, où se cristallisent les acquis fondamentaux de l'analytique, que l'on pourra découvrir les informations essentielles relatives au lieu d'une fondation radicale de l'ontologie. Il s'agira alors de décider de la signification historique d'une telle expérience fondatrice : quel est le lieu d'une histoire de l'Être réconciliée avec elle-même, c'est-à-dire accomplie dans la vie authentique ? Est-il permis au Dasein d'atteindre en solitaire cet état de la réconciliation?

\section{La position du problème de l'Être}

À l'élaboration concrète de la question du sens de l'Être, projet auquel est initialement consacrée la rédaction de Sein und Zeit, le temps ne répond que dans un but provisoire, en tant qu'horizon général de sa compréhension ${ }^{1}$. Vécue comme temporalité, c'est cette compréhension qui rend possible, en même temps qu'elle le détermine, le comportement du Dasein - celui qui questionne dans la Seinsfrage - envers l'étant, « qu'il s'agisse de lui-même, d'autrui ou de l'étant disponible ou subsistant $»^{2}$. L'Être est ce qui détermine l'étant comme étant : il est ce par rapport à quoi l'étant est compris ${ }^{3}$ - bien que lui-même, ainsi que le précise à de nombreuses reprises Heidegger, ne soit ni un étant, ni Dieu, ni le fondement du monde ${ }^{4}$.

${ }^{1}$ Martin Heidegger, Sein und Zeit (désormais cité SuZ), Tübingen, Max Niemeyer Verlag, $1953^{7}$, p. 1 ; trad. fr. É. Martineau, Etre et temps, Paris, Éditions Authentica, édition numérique hors-commerce, p. 21. Je suis ici la pagination de l'édition en ligne, disponible sur http://www.oocities.org/nythamar/etretemps.pdf

2 Martin Heidegger, Gesamtausgabe, Bd. 24 : Die Grundprobleme der Phänomenologie (désormais cité GA 24), F.-W. von Herrmann (Hrsg.), Frankfurt a/M., V. Klostermann, 1975, p. 453 ; trad. fr. J.-F. Courtine, Les problèmes fondamentaux de la phénoménologie, Paris, Éditions Gallimard, coll. "Bibliothèque de Philosophie/ Euvres de Martin Heidegger », 1985, p. 382.

${ }^{3}$ SuZ, § 2, p. 6 ; trad. fr., p. 27. On consultera également SuZ, § 12, p. 53 ; trad. fr., p. 62. Sur la différence ontologique, je renvoie à $G A 24$, p. 453 sq.; trad. fr., p. 382 sq.

${ }^{4}$ Martin Heidegger, «Brief über den Humanismus» (1946), dans Id., Gesamtausgabe, Bd. 9: Wegmarken (désormais cité Brief über den Humanismus), F.- 
Cette interprétation du Dasein comme temporalité (Zeitlichkeit), qui s'élabore progressivement dans l'analytique existentiale, n'est toutefois que la première étape, provisoire, d'une interprétation plus originelle de l'Être, qu'elle a précisément pour tâche de préparer : «Avec cette interprétation du Dasein comme temporalité, souligne Heidegger, n'est pas apportée déjà, et du même coup, la réponse à la question directrice, laquelle porte sur le sens de l'Être en général ${ }^{1}$. « Ce à partir de quoi le Dasein en général comprend et explicite silencieusement quelque chose comme l'Être, poursuit-il, est le temps $»^{2}$, mais le temps n'est que l'horizon d'une telle explicitation, nullement son aboutissement. La découverte de la thèse selon laquelle la temporalité est le mode d'être propre du Dasein ou, plus exactement, sa connexion originaire à l'Être, ne dévoile pas encore la temporalité propre de l'Être, conçue comme Temporalität. Le but ultime de l'ontologie fondamentale, à l'intérieur de laquelle se construit l'analytique du Dasein, est la compréhension concrète du sens de l'Être (Temporalität des Seins), non pas seulement de celui qui l'éprouve en tant qu'Être (sur le mode de la Zeitlichkeit) - bien qu'il faille, en toute logique, commencer la recherche par l'élucidation du mode d'être spécifique de cet étant.

Comme on le constate, le projet de l'ontologie fondamentale et, en son sein, celui de l'analytique existentiale, n'est pas exclusivement descriptif : il ne se limite pas à présenter un aperçu des caractères ontologiques fondamentaux du Dasein. Pour le dire vite, Sein und Zeit a essentiellement pour tâche de préparer le passage de la temporalité du Dasein à celle de l'Être. L'ouvrage de Heidegger poursuit de la sorte une visée prescriptive : l'atteinte du sens concret de l'Être ne doit pas être le seul fait de la recherche philosophique, elle inclut également en elle le projet d'existence du Dasein. À suivre cette interprétation, Sein und Zeit peut tout à fait être considéré comme un ouvrage initiatique à la lecture duquel, comprenant que le Dasein est à chaque fois sien (Jemeinigkeit), le lecteur aura pour tâche de délaisser l'inauthenticité (Uneigentlichkeit) de l'existence qui aura été jusqu'à présent la sienne, et de se mettre en quête de son authenticité (Eigentlichkeit). Ce processus d'initiation du Dasein se trouve inscrit dans la définition que Heidegger donne au $\S 9$ de celui-ci : «L"“essence" [Wesen] de cet étant [le

W. von Herrmann (Hrsg.), Frankfurt a/M., V. Klostermann, 1976, p. 331 ; trad. fr. R. Munier et alii, "Lettre sur l'humanisme», dans Id., Questions III \& IV, Paris, Éditions Gallimard, coll. « Tel», 1990, p. 88.

${ }^{1}$ SuZ, $\S 5$, p. 17 ; trad. fr., p. 35.

${ }^{2}$ Idem. 
Dasein $]$ repose dans son avoir-à-être $[Z u$-sein $] »{ }^{1}$. Parce qu'elle l'informe de ce qu'il a à être, la réponse à la question du sens de l'Être offre au Dasein une éthique - l'Être est lui-même cette éthique ${ }^{2}$.

Dans cette perspective, l'analytique existentiale est foncièrement à la recherche d'une expérience singulière qui rendrait possible ce passage de la temporalité du Dasein à celle de l'Être. La question du sens de l'Être est par conséquent subordonnée à la question qui porte sur la nature de cette expérience en laquelle son sens se révèle. Quelle est alors la nature de cette « expérience fondatrice»?

Comme cela est désormais bien connu, Heidegger, après avoir présenté dans un premier temps l'idée que l'expérience de l'angoisse (Angst) pourrait être une telle expérience $(\S 40)$, en radicalise encore la portée en la désignant comme l'expérience de l'angoisse devant sa propre mort (dont l'analyse culmine aux $\S \S 50-53$ ). C'est grâce à cette expérience d'un type particulier, celle de l'angoisse devant sa propre mort, que le Dasein réalise la connexion de la Zeitlichkeit à la Temporalität.

\section{L'Être et le Dasein}

Bien que l'analytique existentiale ambitionne d'amener graduellement le Dasein en vue de l'Être, il est important de préciser, pour une bonne entente $\mathrm{du}$ projet de Heidegger dans Sein und Zeit, que l'Être en question ne s'identifie pas exclusivement à l'être du Dasein, au sens d'un génitif subjectif. Il s'agit, dans un souci de complémentarité, de comprendre l'être du Dasein au sens d'un génitif objectif: l'Être est la dimension englobante de sens à l'intérieur de laquelle le Dasein existe. Il y a «transpropriation» (Vereignung) de l'être du Dasein et de l'Être de l'étant. C'est dans cette perspective que l'on peut interpréter le $\S 43 \mathrm{c}$ de Sein und Zeit: "C'est seulement aussi longtemps que le Dasein est, autrement dit, aussi longtemps qu'est la possibilité ontique de la compréhension de l'Être, qu' iil y a" de

${ }^{1}$ SuZ, $\S 9$, p. 42 ; trad. fr., p. 54.

${ }^{2}$ La Lettre sur l'humanisme se fera pleinement l'écho d'une telle exigence : « Là où l'essence de l'homme est pensée de façon [...] essentielle, c'est-à-dire à partir uniquement de la question portant sur la vérité de l'Être, mais où pourtant l'homme n'est pas érigé comme centre de l'étant, il faut que s'éveille l'exigence d'une intimation qui le lie, et de règles disant comment l'homme, expérimenté à partir de l'ek-sistence de l'Être, doit vivre conformément à son destin. [...] À cet établissement d'une éthique, nous devons donner tous nos soins » (Brief über den Humanismus, p. 353 ; trad. fr., p. 114). 
l'Être $»^{1}$. La Lettre sur l'humanisme, dans une formule très brève qui résume, en même temps qu'elle la complète, cette citation de Sein und Zeit, insistera à nouveau sur ce chiasme de l'être du Dasein et de l'Être de l'étant : «Il n'y a d'Être qu'autant qu'est l'être-là »². Réciproquement, « l'Être est lui-même dans notre appartenance : car c'est seulement auprès de nous qu'il peut se déployer comme Être, c'est-à-dire être présent $»^{3}$. Un peu plus loin, Heidegger ajoute : «L'homme et l'Être se sont, depuis toujours, déjà atteints l'un l'autre dans leur essence, car c'est en vertu d'une seule et même extension (Zureichung) que tous deux sont transpropriés l'un à l'autre ${ }^{4}$.

Qu'il y ait identité de l'essence du Dasein et de l'essence de l'Être, cela ne doit cependant pas nous faire oublier qu'au sein de cette unité il existe une divergence, une tension : ce que le Dasein " a à être », précisément parce qu'il a à l'être, il ne l'est jamais tout à fait. Quoique le Dasein ne se rapporte à l'étant que selon une modalité déterminée de compréhension de l'Être, cette ouverture ne garantit pas qu'il existe en adéquation avec ce qu'il a à être. Bien au contraire, l'existence du Dasein est le plus souvent vécue sur un mode impropre, en désaccord avec ce qu'il devrait être. C'est la raison pour laquelle la question du sens de l'Être est celle de la compréhension de l'appartenance à la « dimension ontologique » comme modalité de la relation spécifique entre l'Être et le Dasein, en tant que, sur la base de cette compréhension $^{5}$, le Dasein se résout à prendre en charge son «Là », son ouverture à l'Être qu'il a à être $(Z u \text {-sein })^{6}$.

${ }^{1}$ SuZ, $\S 43$ c, p. 212 ; trad. fr., p. 172 . On peut par ailleurs mettre en lien cette proposition essentielle avec celle-ci : «Si nul DASEIN n'existe, nul monde n'est pas non plus "Là" » (SuZ, § 69c, p. 365 ; trad. fr., p. 277).

${ }^{2}$ Brief über den Humanismus, p. 336 ; trad. fr., p. 95.

${ }^{3}$ Martin Heidegger, «Identité et différence », dans Id., Questions I \& II, trad. fr. J. Beaufret et alii, Gallimard, coll. «Tel», 1996, p. 267. Sur le problème de l'identité et de la différence de l'essence de l'homme et de l'essence de l'Être, on consultera J. Pieron, Pour une lecture systématique de Heidegger. Identité, différence, production immanente, Bruxelles, Éditions Ousia, 2010.

${ }^{4}$ Martin Heidegger, «Identité et différence », art. cit., p. 267. C'est moi qui souligne.

${ }^{5}$ Sur le sens «actif» de la compréhension, $c f . S u Z$, $\S 68$, p. 336 ; trad. fr., p. 258 : "Comprendre signifie : être-projetant pour un pouvoir-être en vue de quoi le Dasein existe à chaque fois ».

${ }^{6}$ Au sens de l'« Entschlossenheit ». Cf. SuZ, §60, p. 299 ; trad. fr., p. 233 et $\S 65$, p. 323 sq.; trad. fr., p. 250 sq. À nouveau, par souci de clarté, je ne reviens pas sur l'analyse heideggérienne de la résolution, ainsi que sur l'examen de la temporalité comme sens ontologique du souci, où la résolution est pleinement comprise comme « devançante » (vorlaufende Entschlossenheit). 
Comme nous le verrons plus loin, cette compréhension n'est pas à interpréter comme un acte cognitif: il s'agit essentiellement, dans le chef de Heidegger, d'appréhender celle-ci comme une expérience déterminée. La pleine convergence ou, du moins, la tentative de celle-ci, entre l'essence du Dasein et l'essence de l'Être - entre l'être et l'Être - est le produit d'une expérience vécue : l'angoisse devant sa propre mort. Or, si le Dasein ne peut se résoudre « pour l'existant de fait qu'il est ${ }^{1}{ }^{1}$ que dans l'instant d'une telle expérience, on peut alors légitimement s'interroger sur la nature de cette expérience fondatrice. L'authenticité que le Dasein doit gagner ne risque-telle pas de demeurer un titre vide, une idée fictive ${ }^{2}$, tant que ne sont pas déterminées spécifiquement quelles sont les possibilités vers lesquelles le Dasein, les comprenant dans cette expérience de l'angoisse devant la mort, doit se décider? En bref, "à quoi [le Dasein] doit-il [substantiellement] se décider $»^{3}$ ? À cette question, le $\S 61$ fait écho : «Qu'est-ce que la mort a de commun avec la situation concrète de l'agir ${ }^{4}$ ?

Je crois que l'indication selon laquelle l'expérience de l'angoisse devant la mort est l'expérience en laquelle se manifeste le sens de l'Être n'est pas une donnée suffisante à la spécification de cette expérience. Selon moi, la question du sens de l'Être, telle qu'elle est posée dans l'analytique existentiale, ne réussira à trouver son aboutissement que dans la détermination exacte de la nature de celle-ci. Par suite, cette spécification permettra d'accéder à une élaboration concrète de la question du sens de l'Être, que l'on peut comprendre, selon une indication fondamentale de Sein und Zeit, comme une interprétation plus solide et stable du phénomène de la temporalité ${ }^{5}$. Quelle est enfin cette expérience décisive, qui délivre un sol stable pour l'obtention de la réponse à la question du sens de l'Être ? Comment permettra-t-elle, selon le «système des temps» élaboré par Heidegger, d'articuler la temporalité du Dasein et celle de l'Être?

\section{3. Être-pour-la-mort et histoire de l'Être}

${ }^{1}$ Françoise Dastur, Heidegger et la question du temps, Paris, PUF, coll. «Philosophies », $2005^{3}$, p. 64.

${ }^{2}$ SuZ, § 60, p. 300 ; trad. fr., p. 234.

${ }^{3} S u Z, \S 60$, p. 298 ; trad. fr., p. 233.

${ }^{4}$ SuZ, § 61, p. 302 ; trad. fr., p. 236.

5 « La thèse qui dit que le sens du Dasein est la temporalité doit se confirmer au contact de la réalité concrète de la constitution fondamentale de cet étant » (SuZ, $\S 61$, p. 302 ; trad. fr., p. 236). 
Cette «élaboration plus concrète de la temporalité ${ }^{1}$ est offerte par l'existential de l'historialité (Geschichtlichkeit). Elle constitue, comme le souligne Heidegger, l'aperçu le plus originaire que l'on puisse avoir de la temporalité ${ }^{2}$ - même si ce n'est que parce que le Dasein est temporel dans le fond de son être qu'il peut exister historialement ${ }^{3}$. L'historialité parvient à comprendre le Dasein encore plus originairement que la temporalité dans le projet de son existence authentique ${ }^{4}$. La spécification de l'expérience fondatrice d'une existence authentique, dont l'esquisse est tracée au $\S 50$, concerne ainsi, au premier plan, l'être historial du Dasein: "L'être authentique pour la mort, c'est-à-dire la finitude de la temporalité, est le fondement retiré de l'historialité $d u$ Dasein $»^{5}$. Or, comment, dans la relation qui le lie à sa propre mort, le Dasein réussit-il à "provenir» (geschehen) pleinement au sein de l'Être? En quoi l'expérience de l'angoisse devant la mort, en laquelle le Dasein éprouve sa finitude, permet-elle d'élaborer concrètement une temporalité vécue comme appartenance à une histoire ?

L'expérience de l'angoisse devant la mort, en laquelle le Dasein reçoit le titre insigne d'être-pour-la-mort (Sein zum Tode), doit délivrer à celui-ci une vue adéquate de son pouvoir-être authentique ; mieux encore : elle doit lui intimer le choix définitif pour celui-ci - là où la seule expérience de l'angoisse (§ 40), levant quelque peu le voile sur les possibilités authentiques du Dasein, les recouvrait tout aussi vite dans le choix pour le retour à une existence inauthentique, sous l'emprise du "On» (das Man). Il peut toutefois sembler paradoxal que Heidegger insiste à ce point sur l'importance de la mort. En effet, Heidegger relève au $\S 74$ l'impossibilité de l'expérience de l'angoisse devant la mort à fournir directement les possibilités sur lesquelles se fonde une existence authentique :

\footnotetext{
${ }^{1}$ SuZ, $\S 74$, p. 382 ; trad. fr., p. 288.

${ }^{2}$ SuZ, § 66, p. 332 ; trad. fr., p. 255.

${ }^{3}$ SuZ, $\S 72$, p. 376 ; trad. fr., p. 285 et $\S 76$, p. 396 ; trad. fr., p. 297. C'est parce qu'il est un être temporel que le Dasein peut devenir un être historique : "L'analyse de l'historialité $d u$ Dasein tente de montrer que cet étant n'est pas "temporel" parce qu'il "est dans l'histoire", mais au contraire qu'il n'existe et ne peut exister historialement que parce qu'il est temporel dans le fond de son être » $(\mathrm{SuZ}, \S 72$, p. 376 ; trad. fr., p. 285). On consultera également Heinz Dieter Kittsteiner, MarxHeidegger. Les Philosophies gnostiques de l'histoire, trad. fr. É. Prokob, Paris, Éditions du Cerf, coll. « Passages », 2007, p. 107.

${ }^{4}$ SuZ, $\S 72$, p. 372 ; trad. fr., p. 282.

${ }^{5}$ SuZ, § 74, p. 386 ; trad. fr., p. 291.
} 
Nous devons nous demander d'où en général peuvent être puisées les possibilités vers lesquelles le Dasein se projette facticement. La marche ferme et tenace (Das vorlaufende Sichentwerfen) vers la possibilité indépassable de l'existence, la mort, garantit seulement la totalité et l'authenticité de la résolution. Cependant, les possibilités facticement ouvertes de l'existence ne sauraient être empruntées à la mort, et cela d'autant moins que le devancement vers la possibilité ne signifie point une spéculation sur celle-ci, mais justement un retour vers le Là-factice (das faktische $D a)^{1}$.

Au $\S 72$, Heidegger avait présenté les raisons de cette impossibilité d'emprunter directement les possibilités à la mort :

La mort n'est pourtant que la «fin » du Dasein; ou, pour le dire formellement, elle est seulement l'une des fins qui circonscrivent la totalité du Dasein. Or l'autre « fin », c'est le « commencement», la « naissance». Seul l'étant qui se trouve « entre » naissance et mort représente le tout cherché ${ }^{2}$.

Tantôt l'angoisse devant la mort est célébrée, car elle est la seule expérience en laquelle le projet d'une existence authentique se révèle au Dasein, tantôt elle semble impuissante à indiquer précisément le chemin vers une telle existence, du fait de la vacuité constitutive de l'horizon auquel elle ouvre. Selon le témoignage qu'apporte cet extrait du $\S 72$, il est néanmoins possible de surmonter cette dualité : la mort, comprise authentiquement comme fin de l'existence, doit engager le Dasein sur le chemin du retour vers le lieu de sa naissance. L'être-pour-la-mort (Sein-zum-Tode) est donc bien plutôt, selon la remarque de Heidegger dans une lettre à Hannah Arendt ${ }^{3}$, un «être-en-vuede-la-mort » qui, l'ayant effectivement en vue, se décide pour sa propre vie ${ }^{4}$. Dans l'expérience de l'angoisse devant la mort, le Dasein fait retour vers son « Là-factice », qu'il apporte toujours nativement avec lui ${ }^{5}$ — car le Dasein factice existe nativement, et c'est nativement encore qu'il meurt au sens de l'être en vue de la mort ${ }^{6}$.

${ }^{1}$ SuZ, § 74, p. 383 ; trad. fr. (modifiée), p. 289.

${ }^{2}$ SuZ, § 72, p. 372-373 ; trad. fr., p. 282.

${ }^{3}$ Hannah Arendt \& Martin Heidegger, Lettres et autres documents (1925-1975), trad. fr. P. David, Paris, Éditions Gallimard, 2001, p. 139, cité dans Pascal David, «Tempus mortis », dans Maxence Caron (dir.), Heidegger, Paris, Éditions du Cerf, coll. «Les Cahiers d'Histoire de la Philosophie », 2006, p. 264-265.

${ }_{5}^{4}$ Pascal David, « Tempus mortis », art. cit., p. 265.

5 «Le Dasein, dit Heidegger, apporte nativement (von Hause) avec lui son Là » (SuZ, § 28, p. 133 ; trad. fr., p. 119).

${ }^{6}$ SuZ, $\S 72$, p. 374 ; trad. fr., p. 283. 
L'être-pour-la-mort se révèle être originairement un être-pour-lanaissance $^{1}$. En retrouvant le lieu de ses origines, le Dasein rencontre plus qu'un simple endroit spatialement déterminé. Lorsqu'il retourne à sa terre natale, le Dasein comprend qu'il appartient à un monde et qu'il existe facticement avec d'autres - en tant que ce monde est toujours déjà un monde commun. Sous le titre plutôt vague de «monde» se cache donc une réalité concrète. Pour appréhender celle-ci, on peut se rappeler ce qu'affirme Heidegger au sujet de la structure ontologique fondamentale de l'être-dansle-monde (In-der-Welt-sein). S'appuyant sur l'étymologie de la préposition 《in- » (dans), Heidegger définit l'être-dans-le-monde comme « séjourner », « habiter». Etre-dans-le-monde signifie par conséquent «habiter auprès de... », « être familier de... $»^{2}$, la préposition «in-» désignant cette relation de proximité, qui n'est aucunement réductible à une quelconque relation spatiale $^{3}$. Ce qu'il s'agit de penser, c'est la co-appartenance du Dasein et du monde auquel il est jeté (Geworfenheit), qui n'est pas une détermination locale. En outre, cette structure fondamentale de l'être-dans-le-monde, qui n'est pas identifiable non plus à un mode d'être auprès de l'étant, la facticité (Faktizität), est toujours déjà ce que je partage avec les autres : le monde du Dasein est monde commun (Mitwelt) ${ }^{4}$. Heidegger dira même que « le monde est aussi Dasein $»^{5}$. Étant jeté, le Dasein est assigné à un monde et il existe facticement avec d'autres ${ }^{6}$. Le monde délivre au Dasein un projet d'existence (Gewurf) qui lui est propre, mais qu'il doit aussi faire sien; mieux : l'accomplir.

Comme je l'ai indiqué en introduction, c'est de savoir quoi accomplir (et peut-être aussi comment) dont il est question dans les paragraphes sur l'historialité. L'historialité est en effet le mode selon lequel le Dasein vit ses « origines » (Ursprünge) ou, pour le formuler différemment, elle est le mode

1 À proprement parler, il ne s'agit pas d'une phénoménologie de la «natalité » (Gebürtigkeit), ainsi que le soutient Jean Greisch, en associant à ce $\S 72$ un célèbre extrait de la Condition de l'homme moderne de Hannah Arendt (cf. Jean Greisch, Ontologie et temporalité. Esquisse d'une interprétation intégrale de Sein und Zeit, Paris, PUF, coll. «Épiméthée », 1994, p. 356). La natalité n'est pas un existential. Elle est le moment où l'homme, jeté dans le monde, devient Dasein — où il naît Dasein.

${ }^{2}$ SuZ, § 12, p. 54 ; trad. fr., p. 63.

3 Comme le fait très judicieusement remarquer Jean Greisch, Ontologie et temporalité. Esquisse d'une interprétation intégrale de Sein und Zeit, op. cit., p. 123.

${ }^{4}$ SuZ, $\S 26$, p. 118 ; trad. fr., p. 109.

${ }^{5}$ Idem.

${ }^{6} \mathrm{SuZ}, \S 74$, p. 383 ; trad. fr., p. 289. 
d'être temporel en lequel le Dasein " advient ${ }^{1}$, et sur la base duquel la participation à une histoire du monde devient possible. L'historialité désigne la participation ( $\mu \varepsilon \dot{\varepsilon} \theta \varepsilon \xi 1 \varsigma)$ à l'histoire, qui se fonde précisément sur une appropriation particulière (i.e. authentique ou inauthentique) des possibilités délivrées par le Là-factice. Le Dasein conquiert son authenticité dès qu'il se décide à faire retour vers celui-ci, c'est-à-dire le monde auquel il est assigné ${ }^{2}$ - car, "jeté, le Dasein est remis à lui-même et à son pouvoir-être, mais cependant en tant qu'être-dans-le-monde $»^{3}$.

Les possibilités factices de l'être-jeté, qui sont léguées au Dasein parce qu'il est un tel être-jeté, sont celles d'un héritage que le Dasein, en tant qu'il appartient, avec sa génération, à une tradition, doit assumer ${ }^{4}$. Dans ce geste de liberté qu'est le choix d'une existence authentique, le Dasein se résout à prendre en charge les possibilités traditionnelles de sa «terre natale»:

La résolution où le Dasein revient vers lui-même ouvre les possibilités à chaque fois factices d'exister authentique à partir de l'héritage qu'elle assume en tant que jetée. Le retour résolu vers l'être-jeté abrite en soi un sedélivrer [Sichüberliefern] des possibilités traditionnelles, quoique non pas nécessairement en tant que traditionnelles ${ }^{5}$.

\footnotetext{
${ }^{1}$ Du verbe advenir : geschehen.

${ }^{2}$ «La résolution anticipante [vorlaufende Entschlossenheit], qui est le mode d'être authentique du Dasein implique [...] nécessairement la reprise [Wiederholung] de possibilités trouvées dans l'être-jeté [Geworfenheit] et donc léguées par le Dasein passé dans la projection de son propre avenir » (Jacques Rivelaygue, «Le problème de l'histoire dans Etre et Temps », dans Jean-Pierre Cometti \& Dominique Janicaud (dir.), "Etre et Temps » de Martin Heidegger. Questions de méthode et voies de recherches, s.l., Éditions Sud, 1989, p. 265).

${ }^{3}$ SuZ, § 74, p. 383 ; trad. fr., p. 289.

4 «L'historialité désigne le fait que l'insertion du Dasein dans une histoire (collective) appartient à son être même et le définit. Aussitôt qu'un Dasein vient au monde, il est inséparable d'une "génération" à laquelle il appartient, et de ce fait il a des prédécesseurs et des héritiers. [...] Cette appartenance le qualifie [le Dasein] en son être » (Marlène Zarader, Lire Être et temps de Heidegger, Paris, Librairie philosophique J. Vrin, coll. "Bibliothèque d'Histoire de la Philosophie», 2012, p. 73).

${ }^{5}$ SuZ, § 74, p. 383 ; trad. fr., p. 289. Pour le dire vite, ces possibilités traditionnelles qui, en fait, ne le sont pas, peuvent être interprétées dans le sens d'un dévoiement de la tradition, par exemple de ce que l'on appelle le folklore, et qui ne désigne plus, ou seulement de très loin, l'ensemble des manifestations culturelles propres à une communauté déterminée.
} 
Ce que découvre le Dasein, dans ce mouvement en retour que constitue l'expérience de l'angoisse devant la mort, est la tradition à laquelle il appartient. De la sorte, le lieu de la naissance n'est pas de facto l'aboutissement du parcours du Dasein. Parce qu'il scelle originairement l'appartenance de celui-ci à la tradition, le lieu de sa naissance est chargé de communiquer au Dasein la signification légitime de cette appartenance. Déjà dans l'important $\S 6$, qui porte sur «La Tâche d'une destruction de l'histoire de l'ontologie », Heidegger faisait référence à la tradition qui recouvre d'abord et le plus souvent ce qu'elle « transmet ${ }^{1}$. Ici, au $\S 74$, dans l'expérience qui est en jeu, la tradition peut enfin pleinement délivrer l'héritage en lequel elle existe concrètement. La tradition recouvre alors intégralement un caractère positif, d'authenticité, un caractère qu'avait laissé apercevoir le $\S 6^{2}$.

En dépit de sa primauté, la tradition n'est pas la fin de la quête du Dasein. Au moment où le Dasein se délivre résolument une possibilité héritée (et néanmoins choisie), il aperçoit infiniment plus qu'une tradition :

La finitude saisie de l'existence [l'être en vue de la mort] arrache à la multiplicité sans fin des possibilités immédiatement offertes de la complaisance, de la légèreté, de la dérobade [auxquelles on peut ajouter le contenu que livre avec évidence la tradition ${ }^{3}$ ] et transporte le Dasein dans la simplicité de son destin [seines Schicksals] ${ }^{4}$.

\section{4. Être-pour-la-mort et destin}

La tradition n'est pas le dernier mot de l'existence authentique du Dasein : en elle repose son destin. L'existential du destin était déjà présenté au $\S 12$, en tant qu'il est compris dans le caractère ontologique fondamental de la facticité, entendu comme relation intime avec l'être-dans-le-monde : «Le concept de facticité inclut ceci: l'être-dans-le-monde d'un étant "intramondain", mais d'un étant capable de se comprendre comme lié en son "destin" [Geschick] à l'être de l'étant qui lui fait encontre à l'intérieur de son propre monde $»^{5}$. L'être-dans-le-monde étant essentiellement celui d'un

${ }^{1}$ SuZ, § 6, p. 21 ; trad. fr., p. 38.

${ }^{2}$ Bien que la tradition recouvre le plus souvent ce qu'elle transmet, c'est elle qui possède néanmoins le rôle de la transmission ( $c f . S u Z, \S 6$, p. 21 ; trad. fr., p. 38).

${ }^{3} \mathrm{Idem}$. Sur la tradition et, plus généralement sur le « On » (Man), je renvoie à SuZ, $\S 6$ et $\S 27$.

${ }^{4} S u Z, \S 74$, p. 384 ; trad. fr., p. 289.

${ }^{5} \mathrm{SuZ}, \S 12$, p. 56 ; trad. fr. (modifiée), p. 64. 
monde commun, on peut en déduire que c'est à l'être-pour-autrui que le destin doit renvoyer le Dasein. C'est la raison pour laquelle Heidegger, en introduisant le caractère destinal de l'être-dans-le-monde, parle du destin comme Geschick et non comme Schicksal : en utilisant le terme Geschick, il s'agit d'insister sur l'élément commun de cette destinée - comme souvent, chez Heidegger, avec l'emploi du préfixe «Ge-», que l'on retrouvera, par exemple, dans les concepts de Gestell et de Geviert.

La dimension ontologique à l'intérieur de laquelle se déploie l'êtrepour-autrui, indéfectiblement lié au monde sur lequel le Dasein, existant, est jeté, Heidegger la nomme, au §74, «communauté » (Gemeinschaft) ou «peuple» (Volk) :

Mais si le Dasein destinal [au sens de «Schicksal»] comme être-dans-lemonde existe essentiellement dans l'être-avec-autrui, son advenir est un advenir commun [Geschick], il est déterminé comme destin commun, terme par lequel nous désignons l'advenir [Geschehen] de la communauté [Gemeinschaft $]$, du peuple $[\text { Volk }]^{1}$.

Ce destin commun n'est pas la somme des destins individuels, pas plus que l'être-ensemble (Miteinandersein), comme Heidegger l'expose aux $\S \S 25-26$, n'est la somme de plusieurs sujets ${ }^{2}$. Il s'agit de comprendre ce destin commun mais aussi, plus généralement, la communauté (Gemeinschaft) en laquelle et par laquelle il se déploie, comme l'unité (Sammlung) d'une appartenance mutuelle, fondée sur la tradition ${ }^{3}$. En ce sens, le mouvement d'appropriation de la tradition n'est pas la simple récupération de celle-ci, la tradition exige bien plus que cela du Dasein : la participation à un destin commun. La destinée singulière - singulière parce qu'elle s'affirme dans la compréhension de sa plus intime finitude -, ne se satisfait pas d'elle-même ; elle réclame, pour atteindre son unicité, que le Dasein concoure à une destinée commune, c'est-à-dire qu'il prenne en charge son propre destin en tant qu'il s'agit d'un destin commun.

${ }^{1}$ SuZ, § 74, p. 384 ; trad. fr. (modifiée), p. 290. Cf. Brief über den Humanismus, p. $337-338$; trad. fr., p. 96.

${ }^{2}$ "La clarification de l'être-dans-le-monde a montré que ce qui "est" de prime abord n'est point un simple sujet sans monde, et que rien de tel n'est non plus jamais donné. Et en fin de compte, tout aussi peu est donné de prime abord un Moi isolé sans les autres » (SuZ, § 25, p. 116 ; trad. fr. (modifiée), p. 107-108) et « Le monde du Dasein est monde commun [Mitwelt]» (SuZ, § 26, p. 118 ; trad. fr., p. 109).

${ }^{3} C f$. Martin Heidegger, Einführung in die Metaphysik, Tübingen, Max Niemeyer Verlag, 1953, p. 102. 
La répétition (Wiederholung) de ce qui est toujours déjà donné dans le sillage de la tradition - «Die Wiederholung ist die ausdrückliche Überlieferung ${ }^{1}$ - est le mode selon lequel le Dasein existe expressément comme destin. Ce n'est que dans la répétition que le Dasein, en assumant son attachement à l'héritage transmis - au « répétable » - s'ouvre au destin qui l'attend et, dans cette ouverture, se tient prêt à l'accomplir :

Nous caractérisons la répétition comme le mode de la résolution auto-délivrante par lequel le Dasein existe expressément comme destin. [...] L'autodélivrance devançante, contenue dans la résolution, au Là de l'instant, nous la nommons le destin. En lui se fonde conjointement le co-destin, terme par lequel nous entendons le provenir du Dasein dans son être-avec-autrui ${ }^{2}$.

L'expérience fondatrice doit par conséquent être telle qu'elle ouvre le Dasein à la dimension collective de son existence authentique. L'expérience de l'angoisse devant sa propre mort, qui transporte le Dasein dans l'horizon d'un destin commun, est, de ce fait, une expérience commune.

\section{L'expérience fondatrice du combat}

Quoique l'analytique existentiale soit fondamentalement incapable d'élucider de façon exacte ce pour quoi le Dasein se décide, dans la résolution, à chaque fois facticement - mais comment le pourrait-elle, sinon sans perdre la généralité qui caractérise le propos philosophique ? - , Heidegger donne cependant des indications relativement précises quant à savoir où et comment le Dasein peut accéder à sa mobilité historique authentique - entendue désormais comme l'entrée dans un destin commun. Sur la spécification de l'expérience fondatrice, Heidegger semble en effet très clair: c'est dans l'expérience du combat (Kampf), instant où «l'être-homme », au plus proche de ses compagnons, se sent libre pour le sacrifice qu'une telle situation peut exiger de lui $(\S 74)$ :

C'est dans la communication qui partage et dans le combat [In der Mitteilung und im Kampf] que se libère la puissance du destin commun [Geschick]. Le co-destin destinal du Dasein [Das schicksalhafte Geschick des Daseins] dans

${ }^{1}$ SuZ, $\S 74$, p. 385 ; trad. fr., p. 290.

${ }^{2}$ SuZ, § 74, p. 386 ; trad. fr., p. 290-291. 
et avec sa «génération » constitue le provenir (Geschehen) plein, authentique du Dasein ${ }^{1}$.

Un peu plus loin, à la fin du $\S 75$, Heidegger précise le sens d'une telle expérience du combat :

Dans la résolution est contenue la stabilité existentiale qui, par essence, a déjà anticipé tout instant possible né d'elle. La résolution comme destin est la liberté pour le sacrifice, tel qu'il peut être exigé par la situation, d'une décision déterminée ${ }^{2}$.

Heidegger n'est pas très prolixe, dans Sein und Zeit, sur cette expérience du combat et sur l'importance de la compréhension qu'un libre sacrifice de soi peut, à chaque instant, être exigé par la communauté ${ }^{3}$. Peu importe, il n'en reste pas moins que, dans ce sommet du Hauptwerk que constitue le $\S 74$, la communauté, le peuple, le combat et la liberté pour le sacrifice sont clairement associés en tant que constituants de l'expérience fondatrice qui révèle au Dasein la dimension authentique de son existence.

À présent, on voit d'autant mieux comment l'historialité constitue une élaboration plus concrète de la temporalité. Libre pour la mort, c'est-à-dire prêt à mourir, le Dasein assume, au moment du combat, son être-jeté. Dans l'instant de la situation où un sacrifice peut être exigé de lui, le Dasein rassemble, dans le projet de sa résolution, ce que, suivant son être-jeté, il a à être :

Seul un étant qui est essentiellement avenant en son être, de telle manière que, libre pour sa mort et se brisant sur elle, il puisse se laisser re-jeter vers son Là factice, autrement dit seul un étant qui, en tant qu'avenant, est en même temps étant-été, peut, en se délivrant à lui-même la possibilité héritée, assumer son être-jeté propre et être instantané pour «son temps». Seule la temporalité authentique, qui est en même temps finie, rend possible quelque chose comme un destin, c'est-à-dire une historialité authentique ${ }^{4}$.

${ }^{1}$ SuZ, § 74, p. 384 ; trad. fr. (modifiée), p. 290.

${ }^{2}$ SuZ, § 75, p. 391 ; trad. fr., p. 294.

3 « La résolution comme destin est la liberté pour le sacrifice, tel qu'il peut être exigé par la situation, d'une décision déterminée » (SuZ, § 75, p. 391 ; trad. fr., p. 294).

${ }_{4}$ «Nur Seiendes, das wesenhaft in seinem Sein zukünftig ist, so daß es frei für seinen Tod an ihm zerschellend auf sein faktisches Da sich zurückwerfen lassen kann, das heißt nur Seiendes, das als zukünftiges gleichursprünglich gewesend ist, kann, sich selbst die ererbte Möglichkeit überliefernd, die eigene Geworfenheit über-nehmen 
Le temps n'est plus, dans l'expérience du combat, une suite discontinue d'instants ponctuels qui se déroule selon un sens linéaire. Dans l'instant de la lutte, le Dasein ressaisit un passé qui ne se limite pas à sa seule existence singulière mais qui, au contraire, est élargi à l'histoire de sa tradition et, dans cette réappropriation de son héritage, vise à son accomplissement. Passé et futur fusionnent, dans l'instant présent de la résolution, comme une seule dimension homogène qui se réalise dans le moment de l'être-résolu :

La résolution constitue la fidélité de l'exigence envers le Soi-même propre. En tant que résolution prête à l'angoisse, la fidélité est en même temps possible respect de l'unique autorité que puisse avoir un libre exister, c'est-àdire des possibilités répétables de l'existence. Ce serait mécomprendre ontologiquement la résolution que d'imaginer qu'elle n'est effective en tant que « vécu » qu'aussi longtemps que «dure » l'« acte» de décision. [...] La continuité ne se forme pas d'abord par et à partir de l'ajointement d'« instants », mais ceux-ci naissent au contraire de la temporalité déjà é-tendue de la répétition en tant qu'étant-été de façon avenante ${ }^{1}$.

Peut-on décrire plus exactement la nature de l'expérience ontologique fondamentale, qui s'est affirmée comme expérience du combat? Dans son commentaire de La Germanie, l'un des hymnes de Hölderlin auxquels il consacre un cours en 1934-1935, Heidegger revient de façon beaucoup plus précise sur cette expérience du combat. Je me permets de citer un important passage de ce commentaire dans son intégralité, car il est particulièrement éclairant pour notre propos :

Chez les soldats au front, la camaraderie [Die Kameradschaft der Frontsoldaten] ne provient pas d'un besoin de se rassembler parce que d'autres personnes dont on est éloigné ont fait défaut, ni d'un accord préalable [quelque chose comme un contrat] pour s'enthousiasmer en commun; sa plus profonde, son unique raison est que la proximité de la mort [die Nähe des Todes] en tant que sacrifice a d'abord amené chacun à une identique annulation [Nichtigkeit], qui est devenue la source d'une appartenance absolue à chacun des autres. C'est justement la mort que chaque homme doit mourir pour lui seul et qui isole à l'extrême chaque individu, c'est la mort, et l'acceptation du sacrifice qu'elle exige, qui créent avant tout l'espace de communauté dont jaillit la camaraderie. La camaraderie a-t-elle donc sa source dans l'angoisse ?

und augenblicklich sein für "seine Zeit". Nur eigentliche Zeitlichkeit, die zugleich endlich ist, macht so etwas wie Schick-sal, das heißt eigentliche Geschichtlichkeit möglich» (SuZ, § 74, p. 385 ; trad. fr. (légèrement modifiée), p. 290).

${ }^{1}$ SuZ, § 75, p. 391 ; trad. fr., p. 294. 
Oui et non. Non, si, comme le petit-bourgeois [Spiessbürger], on entend par angoisse le tremblement éperdu d'une lâcheté qui a perdu la tête. Oui, si l'angoisse est conçue comme une proximité métaphysique de l'absolu qui n'est accordée qu'à l'autonomie et à l'acceptation suprêmes. Si nous n'intégrons pas de force à notre Dasein des puissances qui lient et isolent aussi absolument que la mort comme sacrifice librement consenti, c'est-à-dire qui s'en prennent aux racines du Dasein de chaque individu, et qui résident d'une façon aussi profonde et entière en tout savoir authentique, il n'y aura jamais de «camaraderie »: tout au plus une forme particulière de société [Gesellschaft $]^{1}$.

On retrouve dans cet extrait les thèmes de la critique de la société (Gesellschaft), courante à l'époque ${ }^{2}$, du contractualisme, de la sollicitude, etc. Dans le cadre de cette étude, seule m'intéresse la scène à laquelle Heidegger fait référence pour illustrer ce que j'ai appelé l'expérience fondatrice: la proximité avec la mort, qui devient liberté pour le sacrifice de sa propre existence, la vie des soldats sur le front, c'est-à-dire l'expérience de la guerre, en offre l'illustration la plus parfaite. Le passage du destin singulier (Schicksal) à la destinée commune (Geschick) s'opère dès lors que chaque individu, ayant fait sien son Dasein dans le libre consentement du sacrifice, se conçoit membre d'une communauté - à laquelle lui ouvre de facto ce consentement au sacrifice de soi. En ce sens, si le destin peut être qualifié de commun, c'est précisément parce qu'il se fonde dans la communauté (Gemeinschaft) et le peuple (Volk), et non pas sur la seule destinée personnelle.

${ }^{1}$ Martin Heidegger, Gesamtausgabe, Bd. 39 : Hölderlins Hymnen "Germanien » und "Rhein» (désormais cité GA 39), S. Ziegler (Hrsg.), Frankfurt a/M., V. Klostermann, 1980, p. 72-73 ; trad. fr. F. Fédier \& J. Hervier, Les Hymnes de Hölderlin : La Germanie et Le Rhin, Paris, Éditions Gallimard, coll. « Bibliothèque de Philosophie/Série Martin Heidegger », 1988, p. 76-77. Pour un intéressant commentaire de cet extrait, je renvoie à Marc Crépon, "Être pour la mort" et "Pensée du nous" », dans Gérard Bensussan \& Joseph Cohen (dir.), Heidegger. Le Danger et la promesse, Paris, Éditions Kimé, coll. « Philosophie en cours », 2006, p. 79-92.

${ }^{2}$ L'opposition Gemeinschaft/Gesellschaft, courante chez Heidegger, était largement présente à l'époque, notamment grâce au célèbre ouvrage de Ferdinand Tönnies, Gemeinschaft und Gesellschaft (1887), dont la deuxième édition, désormais soustitrée Grundbegriffe der reinen Soziologie, parait en 1912, soit deux ans avant la Première Guerre, période durant laquelle l'opposition des deux termes connaîtra une fortune remarquable, notamment dans les milieux conservateurs. 
Le solipsisme existential, qui menaçait l'expérience de l'angoisse ${ }^{1}$, est enfin dépassé, voire sursumé, dans l'expérience commune du combat. Au moment de la lutte sur le front, le Dasein se singularise et, dans cet isolement, entre en communion avec ses camarades autour d'un projet commun ${ }^{2}$. L'angoisse qui caractérise une telle épreuve - le combat à mener - isole le Dasein - car la mort «isole à l'extrême chaque individu» -, mais, paradoxalement, c'est dans ce retour vers la possibilité la plus propre, qu'un espace de communauté jaillit ${ }^{3}$. C'est donc au plus près de la mort, quand il défend avec d'autres un héritage commun, que le Dasein atteint son êtreensemble authentique. Il entre alors pleinement, avec ses compagnons, dans un destin commun qui, toujours déjà le leur, attendait son assomption. Voici enfin résolue, dans l'expérience de la lutte sur le front, la tension entre la possibilité extrême du sacrifice de soi et "la communication qui partage », que Heidegger indiquait au $\S 53$ de Sein und Zeit, à titre de «Projet existential d'un être authentique pour la mort» - et dont les échos se prolongeront jusqu'au $\S 75$ :

Le devancement ouvre à l'existence, en tant que possibilité extrême, le sacrifice de soi et brise ainsi tout raidissement sur l'existence à chaque fois atteinte [...]. Mais la mort, comme possibilité absolue, n'isole que pour rendre, indépassable qu'elle est, le Dasein comme être compréhensif pour le pouvoir-être des autres ${ }^{4}$.

Dans l'expérience de la lutte sur le front, le Dasein se singularise collectivement : non parce qu'il sait qu'il peut mourir avec d'autres - on n'expérimente pas la mort d'autrui —, mais parce que le Soi-même qui est interpellé et qui, dans cet appel, se constitue, est celui d'une communauté qui existe authentiquement au moment de cette expérience commune. L'être du Dasein advient tel le prisme à travers lequel la lumière de l'Être jaillit; cette lumière ne provient pas d'un sujet isolé, elle est celle d'un être-ensemble historique ${ }^{5}$.

\footnotetext{
${ }^{1}$ SuZ, $\S 40$, p. 190 ; trad. fr., p. 158.

${ }^{2}$ L'expérience de la vie sur le front permet de la sorte une sollicitude authentique (Fürsorge). Je ne développerai pas ici cette thématique, $c f . S u Z, \S 26$, p. 122 sq.; trad. fr., p. $111 s q$.

${ }^{3}$ GA 39, p. 73 ; trad. fr., p. 77.

${ }^{4}$ SuZ, § 53, p. 264 ; trad. fr. (modifiée), p. 209-210, cité dans Marc Crépon, « “Être pour la mort" et "Pensée du nous" », art. cit., p. 87.

${ }^{5}$ GA 39, p. 174 ; trad. fr., p. 164.
} 


\section{Conclusion}

La question du sens de l'Être signifie pour le Dasein la possibilité d'atteindre à sa mobilité historique authentique - son existence propre. Cet accès se réalise à la faveur d'une expérience fondatrice, incontestablement commune, et dont j'ai montré que l'illustration paradigmatique semblait être la lutte sur le front : au moment du combat, le Dasein se situe, avec ses compagnons, au plus proche de l'Être. La résolution du problème posé par l'analytique existentiale passe donc par la mise en jeu de l'engagement du Dasein, qu'il soit prêt à vivre une expérience pour laquelle il y va du sacrifice de sa propre vie.

Cette expérience ne constitue néanmoins que le point d'orgue d'une relation entre l'être du Dasein et l'Être de l'étant qui, tout au long de son déploiement, semble plutôt difficile. Ce que j'ai appelé, à la suite de Heidegger, "transpropriation », que l'on pourrait aussi concevoir sous la figure merleau-pontyenne du chiasme, rend justice de cette relation de réciprocité et de co-appartenance entre l'être du Dasein et l'Être de l'étant qui ne parait jamais trouver le repos autrement que dans cet instant paroxystique de la lutte commune. Ainsi, et bien que l'essence du Dasein et l'essence de l'Être convergent, il existe, au sein de cette unité, une divergence, une tension à même l'unité ${ }^{1}$ — l'histoire de l'Être consistant dans le déploiement de cette tension. De ce mouvement tout en systole et diastole en quoi consiste l'histoire de l'Être, on peut déduire qu'à chaque fois que le Dasein s'engagera dans son destin qu'elle touchera à sa fin, car la tension constitutive de celle-ci s'approchera alors de sa résolution. Le dépassement de la Métaphysique comme entrée dans une existence commune authentique signifierait ainsi l'advenue de l'absence d'histoire. Pour Heidegger, l'existence réconciliée avec elle-même effectue comme un saut par-dessus sa propre histoire : elle atteint à cette origine qui s'était découverte au Dasein dans l'expérience fondatrice de la lutte.

Comment toutefois ne pas interpréter un tel saut comme celui du tigre, dont Benjamin parle dans ses thèses «Sur le concept d'histoire $»^{2}$ ? L'histoire de l'Être ne compose-t-elle pas avec une « image "éternelle" du passé », bien plutôt qu'elle ne dépeint «l'expérience unique de la rencontre avec ce

\footnotetext{
${ }^{1}$ À nouveau, je renvoie à Julien Pieron, Pour une lecture systématique de Heidegger. Identité, différence, production immanente, op. cit.

${ }^{2}$ Walter Benjamin, « Sur le concept d'histoire », dans Id., Euvres III, trad. fr. M. de Gandillac et alii, Paris, Éditions Gallimard, coll. « Folio/Essais », 2000, p. 439.
} 
passé $»^{1}$ ? C'est probablement le cas. En pourtant, il faudrait faire en sorte que l'instant de l'angoisse devant sa propre mort soit celui où le passé comme image vraie ressurgit. Mais pour cela c'est l' «instant du danger ${ }^{2}$ lui-même qui doit changer et devenir « assez viril pour faire éclater le continuum de l'histoire $»^{3}$. Alors seulement l'image du passé qui s'offrira inopinément au sujet historique pourra sans atermoiement lui indiquer sa destination, la lutte sur le front se transformant en «combat pour le passé opprimé $»^{4}$.

\footnotetext{
${ }^{1}$ Ibid., p. 441.

${ }^{2}$ Ibid., p. 431.

${ }^{3}$ Ibid., p. 441.

${ }^{4}$ Idem.
} 